SILES / El arte y la creatividad como nuevas formas de bienestar. Primera fase de ARTYS, La Experimental, proyecto de Arte y Salud Comunitaria en la Colonia Experimental de Villaverde Alto (Madrid)

\title{
El arte y la creatividad como nuevas formas de bienestar. Primera fase de ARTYS, La Experimental, proyecto de Arte y Salud Comunitaria en la Colonia Experimental de Villaverde Alto (Madrid)
}

Art and creativity as a new wellbeing ways. The first phase of ARTYS. La Experimental, Art and Community Health in the Colonia Experimental of Villaverde Alto (Madrid)

Silvia SILES. Universidad Complutense de Madrid (España). shivanataraya2@gmail.com

Resumen: El presente artículo tiene como objetivo describir la primera fase del proyecto de investigación ARTYS La Experimental, un proyecto de Arte y Salud comunitaria, que se lleva a cabo en la Colonia Experimental del barrio madrileño de Villaverde Alto. Se trata de una propuesta práctica diseñada durante el proceso por todas las personas implicadas en él. De esta manera, pretende crear nuevas oportunidades para mejorar el bienestar y la salud de un colectivo concreto a través de la creatividad y promover el uso de las prácticas artísticas como vehículo para mejorar la calidad de vida de la vecindad. Además, parte de los preceptos del arte basado en la comunidad, las prácticas artísticas colaborativas, el arte activista, la promoción de la salud comunitaria a través de los activos en salud, la metodología de investigación-acción participativa y la intervención comunitaria. Se trata de la fase inicial de un proceso que tiene como protagonista acciones artísticas en el espacio público, la participación activa y la toma de decisiones colectiva y que ha tenido lugar desde mayo de 2017 y hasta finales de enero de 2018. Por último, se presenta un análisis y una discusión de los resultados obtenidos hasta el momento de la publicación de este texto.

Palabras clave: arte basado en la comunidad, prácticas artísticas colaborativas, salud comunitaria, activos en salud, investigación-acción participativa, espacio público. 
SILES / El arte y la creatividad como nuevas formas de bienestar. Primera fase de ARTYS, La

\begin{abstract}
The purpouse of this paper is to describe the first phase of the research project ARTYS La Experimental, which is an Art \& Community Health Project that took place in La Colonia Experimental, a neighborhood located in Villaverde Alto in the province of Madrid. The practical proposal is designed during the process by the people involved in it. Thus, the project intends to create new opportunities for a collective group to improve their health and wellbeing across the creativity and the artistic practices as a way of increasing the neighborhood's quality of life. The project is based on the community art-based projects, collaborative arts, activist art, community health promote through health assets, action-research participative method and community intervention. This phase of the research process has been developed from May 2017 to the end of January 2018 which includes artistic actions in the public space, active participation and collective decision-making as key protagonists. Lastly, analysis and disscusion of the results achieved to date are written in this publication.
\end{abstract}

Keywords: community art-based projects, collaborative arts, community health, health assets, action-research participative, public space.

\title{
Introducción
}

ARTYS. La Experimental es un proyecto de promoción de la salud comunitaria a través del arte como metodología para facilitar la participación de la ciudadanía e impulsar la mejora de la calidad de vida de la vecindad de la Colonia Experimental de Villaverde Alto.

La praxis de este trabajo se enmarca en los preceptos del arte basado en la comunidad y de las prácticas artísticas colaborativas y tiene como propósito analizar el funcionamiento de los procesos creativos como herramienta para la promoción de la salud comunitaria. Esta concepción comunitaria se entiende como una expresión colectiva de la salud determinada por la interacción de los individuos que pertenecen a dicha comunidad entre sí y con su entorno. Además, sigue el modelo salutogénico de activos en salud que aporta una visión en positivo que fomenta que las comunidades reorienten su mirada al contexto y se centren en aquello que mejora la salud y el bienestar para potenciar, de esta manera, la equidad en salud (Cofiño, et al., 2016, p. 93). Por lo tanto, son las personas las que tienen la capacidad de llevar a cabo acciones por su salud aprendiendo a responder a los retos del ambiente, y pueden ponerlas en común para generar un "patrimonio comunitario". Por consiguiente, la participación ciudadana como derecho comunitario y como agente de salud constituye uno de los ejes fundamentales de esta propuesta.

El trabajo de investigación que se describe constituye una experiencia concreta del proyecto Arte y Salud que se enmarca dentro de la Estrategia Barrios Saludables 
SILES / El arte y la creatividad como nuevas formas de bienestar. Primera fase de ARTYS, La Experimental, proyecto de Arte y Salud Comunitaria en la Colonia Experimental de Villaverde Alto (Madrid)

2016-2019 ${ }^{1}$, del Servicio de Prevención y Promoción de la Salud del Organismo Autónomo de Madrid Salud del Ayuntamiento de Madrid, y de una beca de formación e investigación de tesis doctoral en Bellas Artes, concedida por dicho organismo para la aplicación de metodologías artísticas en los programas de promoción de la salud del Servicio en los Centros Municipales de Salud Comunitaria $(\mathrm{CMSc})^{23}$

Por tanto, en el presente artículo se describe y analiza la primera fase y los resultados de un proceso en construcción a largo plazo, que ha comenzado en 2017 y que continúa desarrollándose en la actualidad con la actividad de un grupo motor que surge tras las acciones realizadas en este periodo. Se pretende, con ello, reflexionar sobre el potencial que tienen los procesos artísticos y creativos como mecanismos para generar oportunidades que proporcionen salud y bienestar a colectivos y comunidades, mediante una red de colaboración multi, inter y transdisciplinar.

\section{Arte basado en la comunidad, las prácticas artísticas colaborativas y el arte activista}

La obra de arte es un macro-signo que puede tomar varias formas de manifestación, así puede ser un objeto, un espacio, una instalación, un proceso, una intervención, pero se trata de un discurso que se construye semióticamente como un conjunto de signos que funcionan gracias a interacciones internas y externas que produce siempre un cambio en el contexto (Radulescu, 2014). De esta manera, el arte se constituye como una plataforma para generar dinámicas de transformación social y de construcción de democrática, en la que debemos salir de los productos y pensar en los procesos (Miyagui, 2014). Esta afirmación lleva a enunciar el concepto de arte basado en la comunidad o arte comunitario, uno de los modelos de prácticas artísticas en los que se basa esta investigación y que Palacios (2009) asocia:

(...) a un tipo de prácticas que buscan una implicación con el contexto social, que persiguen, por encima de unos logros estéticos, un beneficio o mejora social y, sobre todo, que favorecen la colaboración y la participación de las comunidades implicadas en la realización de la obra. Estas prácticas implican una revisión de los

\footnotetext{
1. Estrategia Barrios Saludables 2016-2019. Recuperado de: https://www.madrid.es/UnidadesDescentralizadas/Salud/ PrevencionYPromocion/EspInformativos/Estrategia\%20gente\%20saludable/ficheros/BARRIOS_SALUDABLES_2018.pdf

2. Los Centros Municipales de Salud Comunitaria (CMSc), son centros especializados en la promoción de la salud y en la prevención de las enfermedades y otros problemas de salud cuyo objetivo no es tratar la enfermedad sino que se centran en prevenirla y en ayudar a las personas, los grupos y las comunidades a conseguir condiciones de vida saludables. Recuperado de: http://madridsalud.es/centros-madrid-salud-cms/

3. Este proyecto, Arte y salud, surge de la colaboración, iniciada en 2012, entre la Facultad de Bellas Artes de la Universidad Complutense de Madrid y el Organismo Autónomo Madrid Salud para llevar a cabo un asesoramiento técnico en actividades, prácticas y proyectos conjuntos desde el arte y la promoción de la salud en los Centros Municipales de Salud Comunitaria (CMSc). De manera que, tras 6 años de trabajo conjunto, en 2017, esta colaboración se transforma en una apuesta por la consolidación del profesional de arte en contextos de salud comunitaria por medio de la concesión 3 becas de formación e investigación a estudiantes de doctorado. Recuperado de: https://www.ucm.es/arteysaludproyectos/noticias/silvia-siles,jorge-fernandez-cedena-y-mar-castillejo,-becarios-de-madrid-salud
} 
SILES / El arte y la creatividad como nuevas formas de bienestar. Primera fase de ARTYS, La

conceptos modernistas de artista y de obra de arte. El artista delega parte de sus funciones tradicionales en el grupo y el concepto de obra artística se transforma por su carácter procesual y de intervención social. (p. 199)

En segundo lugar, y en conexión con la anterior definición, encontramos las prácticas artísticas colaborativas, cuyo origen, según Blanco (2016) se remontaría al nacimiento de los happenings de los años 60. En ellos se observaba una serie de elementos comunes que constituyen las características fundamentales de estas prácticas artísticas: un papel multidisciplinar del artista, su compromiso activo con las comunidades y colectivos con los que trabaja, la implicación activa de un "público" que se convierte en coautor de la obra, su rechazo a su estatus de mercancía de arte y el desarrollo de estrategias para prevenir su mercantilización, y sobre todo su capacidad para comprometer al público en una práctica colaborativa capaz de establecer vínculos y redes de trabajo (Blanco, 2016). En el momento actual, este trabajo colaborativo entre artistas, "públicos" y colectivos sociales es una forma de producción cultural en creciente reconocimiento que emerge como una forma de poner en acción las prácticas artísticas en el contexto de procesos sociales amplios, cuestionando, de esta manera, la separación del arte y la vida como dos esferas independientes la una de la otra (Sánchez de Serdio, 2015).

Es por esto que una práctica cultural no puede descontextualizarse de la red sociocultural en la cual se inserta ya que el producto artístico varía independientemente del propósito del artista y del funcionamiento del objeto (Radulescu, 2014). De esta manera, las prácticas artísticas colaborativas y los métodos del arte basado en la comunidad se convierten en base teórica de esta investigación, así como algunos elementos prácticos del activismo artístico, cuya preocupación central es, según Delgado (2013):

la de exaltar los valores de los espacios urbanos de libre concurrencia, cuestionando la voluntad patente de los poderes en orden a exorcizar la amenaza que para su hegemonía supone la acción colectiva en ellos, acelerando al máximo las virtualidades subversivas latentes en la interacción humana ordinaria que se desarrolla en su seno. (p. 69)

Para ello, contempla parámetros como el posicionamiento crítico, la voluntad de interacción con el ámbito social, la vinculación con la especificidad del lugar y el compromiso con la realidad y promueve actividades prácticas que dotarán de un punto de vista alternativo a los sistemas productivos existentes (Delgado, 2013).

Por tanto, en el contexto concreto de este proyecto el arte activista configura las acciones colectivas desarrolladas predominantemente en espacios públicos (Salazar y Olivos, 2014). A causa de ello, se presentan dichos espacios como una prolongación de la vivienda, un lugar de debate y encuentro donde compartir experiencias y llevar a cabo acciones colectivas, porque: 
SILES / El arte y la creatividad como nuevas formas de bienestar. Primera fase de ARTYS, La Experimental, proyecto de Arte y Salud Comunitaria en la Colonia Experimental de Villaverde Alto (Madrid)

las experiencias artísticas en el espacio urbano, evitan los lugares y convenciones tradicionales, sugieren contextos reales o potenciales para el arte, que reflejen la diversidad del contexto social, y se sitúan en la vida cotidiana, en términos de ambiente, espíritu y significado. (Fernández Quesada, 2004, p. 221)

\section{Promoción de la salud comunitaria, salutogénesis y activos en salud}

Por otro lado, se considera fundamental esclarecer de qué se habla cuando se enuncia la promoción de la salud comunitaria. Según López Ruíz y Padilla Vernáldez (2017) se trata de un enfoque colectivista y emancipador en el que se eliminan todos los factores individuales para la construcción de una salud desde lo colectivo, que es resultado de múltiples procesos de socialización, y que está atravesada por un sistema de género. Además, en este planteamiento se considera a la comunidad como un foco generador de salud puesto que los actores sociales no son sujetos pasivos, sino que, juegan un papel fundamental en la construcción de salud colectiva como agentes de cambio.

Esta propuesta está en consonancia con el modelo salutogénico que, en los años 70, Aaron Antonovsky comenzó a desarrollar, afirmando que, "la salud positiva, es una forma de ver las acciones en salud focalizando la mirada hacia aquello que hace que las personas, las familias y las comunidades aumenten el control sobre su salud y la mejoren" (Hernán, Morgan, y Mena, 2010, p. 8). En este sentido:

los profesionales y las personas se comprometerían mutuamente en un proceso de empoderamiento, de modo que el papel de los profesionales sería apoyar y ofrecer opciones que permitan a las personas tomar decisiones bien fundamentadas, siendo conscientes de los principales determinantes de la salud. (Rivera de los Santos, et al, 2011, p 132)

De esta manera, se debe considerar de relevante importancia los activos en salud dentro de este modelo salutogénico, definidos "como cualquier factor o recurso que potencie la capacidad de los individuos, de las comunidades y poblaciones para mantener la salud y el bienestar" (Morgan y Ziglio 2007; Morgan, Davis y Ziglio, citado en Hernán et al., 2010, p. 16).

\section{Metodología}

Los modelos metodológicos utilizados son la intervención comunitaria y la investigación-acción participativa.

\section{Intervención comunitaria}

Por un lado, el modelo de intervención comunitaria es necesario para comprender el planteamiento transdisciplinar de esta investigación. Se trata de una propuesta "política" de interés general y no limitable a comunidades particulares con grandes necesidades o en situaciones críticas, por lo que puede realizarse en cualquier 
SILES / El arte y la creatividad como nuevas formas de bienestar. Primera fase de ARTYS, La

territorio (comunidad). Además, el objetivo principal que persigue es una mejora y modificación de las políticas sociales existentes, en las que prevalece la visión asistencial que "atiende usuarios" y que excluye la posibilidad de un papel activo de la ciudadanía. Por ello, la finalidad de la intervención comunitaria solo puede ser la mejora de las condiciones de vida de la población interesada, siendo la comunidad el elemento fundamental, y asumiendo el principio de que cualquier situación, individual y/o colectiva es mejorable. Por tanto, este modelo metodológico nace de la concepción de la participación en paridad de condiciones como derecho. En términos específicos, se trata de una metodología que hace de la participación el hecho central, por ello, se sitúa de manera decisiva en el terreno de la democracia y, en particular, de la democracia participativa (Marchioni, Morin y Álamo, 2013).

Investigación-acción participativa

Por el otro, la Investigación-Acción participativa constituye el otro pilar fundamental de este trabajo puesto que, se define como un modelo de planificación, ejecución y evaluación de procesos emancipadores de transformación social mediante la participación colectiva en la investigación. Se trata de un enfoque donde las vivencias de las personas y las particularidades del contexto proporcionan las claves para una mayor comprensión de lo que acontece y donde cobra importancia la acción de aquellos sectores que, hasta el momento, eran tenidos en cuenta sólo como objetos pasivos de estudio (Lewin, 2006). De este modo, la producción y elaboración del conocimiento es compartida por el investigador y el investigado, desde un enfoque integrado de leyes generales y situaciones específicas (Lewin, 2006). En resumen, se puede afirmar que se trata de una manera de hacer, flexible, compartida y creativa, ya que, en el proceso colectivo de investigación surgen problemas y necesidades para conseguir la horizontalidad y la colaboración, que deben ser resueltas de forma creativa.

A causa de ello, en el contexto concreto de esta investigación se tuvieron que generar nuevas herramientas como, por ejemplo, un cuaderno de campo colectivo y on-line a través de un grupo de la aplicación Whatsapp que ha permitido compartir un flujo de información a través de texto, imágenes, vídeos y audios a disposición de todos los miembros de la comunidad involucrada en el proyecto.

Desarrollo primera fase de la experiencia. Artys. La Experimental

El desarrollo del trabajo en $A R T Y S$ gira en torno a los siguientes objetivos:

- Impulsar la mejora del bienestar físico, mental y social de los vecinos y las vecinas a través de las prácticas artísticas.

- Promover la unión grupal/vecinal y las buenas relaciones que permitan crear una red de cuidados mutuos y mejorar la convivencia. 
SILES / El arte y la creatividad como nuevas formas de bienestar. Primera fase de ARTYS, La Experimental, proyecto de Arte y Salud Comunitaria en la Colonia Experimental de Villaverde Alto (Madrid)

- Impulsar la participación activa de los miembros de la comunidad a través de las prácticas artísticas colaborativas para generar experiencias positivas en torno a la promoción de la salud (activos en salud).

- Establecer vínculos y relaciones entre las personas y el espacio público que habitan y comparten.

- Promover el acceso democrático al arte y la cultura como agente de desarrollo de comunidades saludables.

De esta manera, para conseguir estos objetivos, se han puesto en marcha una serie de acciones y actividades, durante el año 2017 y principios del 2018 que se desarrollan a continuación (tabla 1).

Tabla 1

Primera fase de ARTYS La Experimental (2017-principios 2018)

\begin{tabular}{|c|c|}
\hline $\begin{array}{l}\text { Formación de la comisión de trabajo y } \\
\text { primer contacto con la comunidad. }\end{array}$ & $\begin{array}{l}\text { Propuesta de proyecto al Espacio Intersectorial } \\
\text { de Participación Comunitaria en Salud de } \\
\text { Villaverde. } \\
\text { Formación de la comisión de trabajo (CMSc, } \\
\text { Servicio de Convivencia Intercultural en } \\
\text { Barrios, Vocal Vecina grupo municipal Ahora } \\
\text { Madrid). } \\
\text { Encuentros Vecinales (2). }\end{array}$ \\
\hline \multirow[t]{5}{*}{$\begin{array}{l}\text { Taller del Tema al lema (collage, publicidad- } \\
\text { contrapublicidad } y \text { artivismo) }\end{array}$} & $\begin{array}{l}\text { Sesión } 1 \\
\text { Conceptualización teórica: collage, publicidad } \\
\text { y contrapublicidad, y arte activista. } \\
\text { "Gafas La Experimental". }\end{array}$ \\
\hline & $\begin{array}{l}\frac{\text { Sesión } 2}{\text { Collage colectivo. }} \\
\text { Elaboración de eslóganes. }\end{array}$ \\
\hline & $\frac{\text { Sesión } 3}{\text { Creación de } 5 \text { carteles. }}$ \\
\hline & $\begin{array}{l}\text { Sesión } 4 \\
\text { Creación de } 5 \text { carteles. }\end{array}$ \\
\hline & $\begin{array}{l}\frac{\text { Sesión } 5}{\text { Confección del recorrido para pegada de }} \\
\text { carteles } \\
\text { Construcción del árbol. } \\
\text { Creación del manifiesto colectivo. }\end{array}$ \\
\hline Acción en el espacio público & $\begin{array}{l}\text { Pegada de carteles. } \\
\text { Lectura pública del manifiesto junto al árbol. } \\
\text { Celebración vecinal. }\end{array}$ \\
\hline Seguimiento carteles e instalación & $\begin{array}{l}\text { Cuaderno de campo colectivo: grupo de } \\
\text { whatsapp. }\end{array}$ \\
\hline
\end{tabular}


SILES / El arte y la creatividad como nuevas formas de bienestar. Primera fase de ARTYS, La

Formación de la comisión de trabajo y primer contacto con la comunidad.

Para poner en marcha el proyecto, en primer lugar, se creó una comisión de trabajo a partir del Espacio Intersectorial de Participación Comunitaria en Salud de Villaverde Alto, formado por diversas entidades que trabajan en el distrito para construir un proyecto común junto a las vecinas y vecinos de la Colonia Experimental. Este espacio era conocedor de las dificultades, malestares y problemas de salud de la Colonia: una situación de viviendas antiguas y en mal estado de conservación que necesita una remodelación integral por la que llevan luchando varias décadas, problemas con la limpieza de los espacios comunes, problemas de convivencia dentro del propio territorio y con el resto del barrio, y una situación de estigmatización social. Para el abordaje de esta situación, la comisión de trabajo antes mencionada, finalmente queda configurada por: el CMSc y del Servicio de Convivencia Intercultural en Barrios, dos entidades que trabajan en el territorio desde hace años; la Vocal Vecina del grupo Municipal Ahora Madrid; y la recién incorporada artista-becaria del proyecto Arte y Salud.

Como resultado de las primeras reuniones de los miembros esta comisión se plantea el espacio público como prolongación de la vivienda, un lugar de debate, de encuentro y reunión donde compartir experiencias y llevar a cabo acciones colectivas que mejoren la calidad de vida de las personas que lo habitan y, por tanto, su salud. Esta idea surge al comprender el potencial que podían tener los espacios interbloque del territorio como activo en salud y como lugar de intervención artística.

De esta manera, se convoca un "Encuentro Vecinal", con el objetivo de tomar el primer contacto con la vecindad, presentar el proyecto y plantear la posibilidad de su puesta en marcha mediante la pregunta ¿qué podemos hacer en común para sentirnos mejor en nuestro barrio?, que ha constituído el hilo conductor de ARTYS. Ante la buena acogida y el primer intercambio de propuestas sobre cómo llevarlo a cabo en la realidad cotidiana, se convoca un segundo encuentro en el mes siguiente para trabajar en torno a las necesidades sentidas por parte de las vecinas y vecinos manifestadas en el primer encuentro, sobre las potencialidades y habilidades de estos para dar respuesta a esas necesidades y cómo transformarlo en una práctica creativa dirigida a mejorar su bienestar.

Taller del Tema al lema (collage, publicidad-contrapublicidad y artivismo)

Para dar continuidad a la experiencia, se estableció una fecha para comenzar la realización de una serie de talleres de carteles de fomento de la convivencia en la Colonia. La idea surge de las vecinas que han decidido participar en el proyecto y está motivada, en primer lugar, por el malestar generado por la falta de cuidado de los espacios públicos por parte de las propias personas que habitan el barrio, y en segundo, por el énfasis que se quiere poner desde la comisión de trabajo en lo común, el desarrollo y bienestar a través de la participación colectiva, social 
SILES / El arte y la creatividad como nuevas formas de bienestar. Primera fase de ARTYS, La Experimental, proyecto de Arte y Salud Comunitaria en la Colonia Experimental de Villaverde Alto (Madrid)

y ciudadana como lugar de encuentro para compartir la responsabilidad cívica (Giménez Romero, 2013). Las 5 sesiones del taller tuvieron lugar entre los meses de noviembre y diciembre:

\section{Sesión 1.}

Se comienza la primera sesión con una conceptualización teórica sobre collage, publicidad y contrapublicidad, y arte activista, mediante una presentación con imágenes de los lenguajes artísticos que serían clave en las siguientes sesiones prácticas del taller. De este modo, el collage constituyó la técnica manual de confección de los carteles, las estrategias de creación de eslóganes publicitarios y su transformación crítica de la contrapublicidad se emplearon para crear los mensajes, y el artivismo o activismo artístico, sirvió para diseñar una acción pública de visibilización del proceso y de las reclamaciones sociales de las participantes.

Además, se realizó un juego, "Las gafas La Experimental, lo verás todo fenomenal”, con el que se pudo empezar a soñar con los cambios deseados en el espacio que habitan y comparten, tomando como referencia la metodología de movilización ciudadana del juego del Oásis del instituto Elos de Brasil, que invita a una comunidad a proyectar y a construir, de forma cooperativa, sus sueños o aspiraciones colectivas, elegidas por la propia comunidad, movilizando talentos y recursos locales. ${ }^{4}$

\section{Sesión 2.}

Para la primera toma de contacto con el collage, se llevó a cabo una obra colectiva a partir de las visiones ideales del barrio que se manifestaron con "Las gafas La Experimental", realizado un ejercicio de transformación de los sueños sobre el barrio a imágenes visuales sobre una reproducción dibujada del espacio real de la Colonia. Además, en esta misma sesión, se crearon las frases y eslóganes de los carteles.

\section{Sesión 3 y 4.}

En esta sesión, una vez elegidos los 5 eslóganes que más encajaban con las necesidades, demandas y la problemáticas concretas de la Colonia, se dio paso a la creación de los carteles que se desarrolló forma colectiva, tanto en la búsqueda de imágenes, como en la toma de decisiones, recorte y pegado de los fragmentos de periódicos y revistas.

\section{Sesión 5.}

Por último, tuvo lugar el diseño de la acción de visibilización del proyecto en el espacio público. En primer lugar, sobre el mapa del barrio, se marcó el recorrido

4. http://www.3sbizkaia.org/Archivos/Documentos/Enlaces/1911_OTS_OTSAILA_2016.pdf 
SILES / El arte y la creatividad como nuevas formas de bienestar. Primera fase de ARTYS, La

para la pegada de los carteles ya impresos, que finalizaría en emplazamiento donde acontecieron los encuentros vecinales. Esta zona constituye un "parking público" para el resto del barrio, por lo que, de este modo, se intentaría "conquistar" algo de espacio a los coches para poder ejercer el derecho ciudadano del uso del espacio público con una instalación artística: un árbol hecho con contenedores negros en el que se pegarían los carteles.

Por último, se decidió que esta acción constituiría la primera presentación pública de ARTYS y, para ello, se creó un manifiesto colectivo que se analizará más adelante en este texto.

Acción en el espacio público

Tras el taller, la acción de arte activista comienza con la pegada de los carteles siguiendo el recorrido marcado en el mapa y finalizando en lugar donde se había instalado el árbol. Alrededor de este, se delimitó el espacio con cuerdas y se colocó un cartel con la pregunta ¿qué podemos hacer en común para sentirnos mejor en nuestro barrio? junto con una caja con papeles, lápices y pinzas de la ropa invitando a los viandantes a interactuar con la obra. También, dicho espacio acotado sirvió como "escenario" para la lectura colectiva del manifiesto (figura 5) como acto de presentación pública del proyecto. Para finalizar la acción, tuvo lugar una pequeña celebración vecinal alrededor de la instalación artística.

\section{Seguimiento carteles e instalación}

Una vez finalizada la acción artística, se considera importante observar las consecuencias de esta en el contexto físico y simbólico en el que se ha llevado acabo. Por ello, se estima imprescindible realizar un seguimiento colectivo de los dispositivos artísticos colocados en la calle durante las semanas o meses posteriores a la acción. Para realizar este seguimiento, se elige un medio digital que ofrece facilidades de interacción entre las personas involucradas en el proyecto: un grupo de la aplicación Whatsapp. A través de él, pudieron participar tanto las personas que habían llevado a cabo la acción directa como vecinos y vecinas que habían asistido al evento, compartiendo fotos, vídeos y audios del estado de los dispositivos artísticos. También, permitió la organización de los miembros del grupo para la conservación de los objetos cuando éstos presentaban algún tipo de deterioro.

\section{Evaluación}

Como cierre de esta primera fase, se realizó una sesión de evaluación de todas y cada una de las partes, elementos y acontecimientos ocurridos durante esta etapa del proyecto. En esta evaluación se analizaron cuestiones como: el lugar en el que se habían realizado los talleres y la acción, la manera en la que se habían difundido las actividades, la participación, el funcionamiento del trabajo colectivo, y el equilibrio en el reparto de tareas. Sin embargo, debido a la información significativa 
SILES / El arte y la creatividad como nuevas formas de bienestar. Primera fase de ARTYS, La Experimental, proyecto de Arte y Salud Comunitaria en la Colonia Experimental de Villaverde Alto (Madrid)

recopilada en el manifiesto colectivo antes mencionado, se considera que esta fue la primera evaluación que se llevó a cabo, por lo que también se incluye en el análisis y discusión de los resultados.

\section{Análisis y discusión de resultados}

Como resultados de la primera fase de esta experiencia se observa que los encuentros vecinales han proporcionado canales comunicativos para expresar demandas y necesidades sentidas por parte de la vecindad en relación a su bienestar y salud integral (figura 1). Además, ha impulsado la creación de respuestas a esas demandas con los propios recursos y habilidades con los que ya cuenta el conjunto de la comunidad (activos en salud) (figura 1).

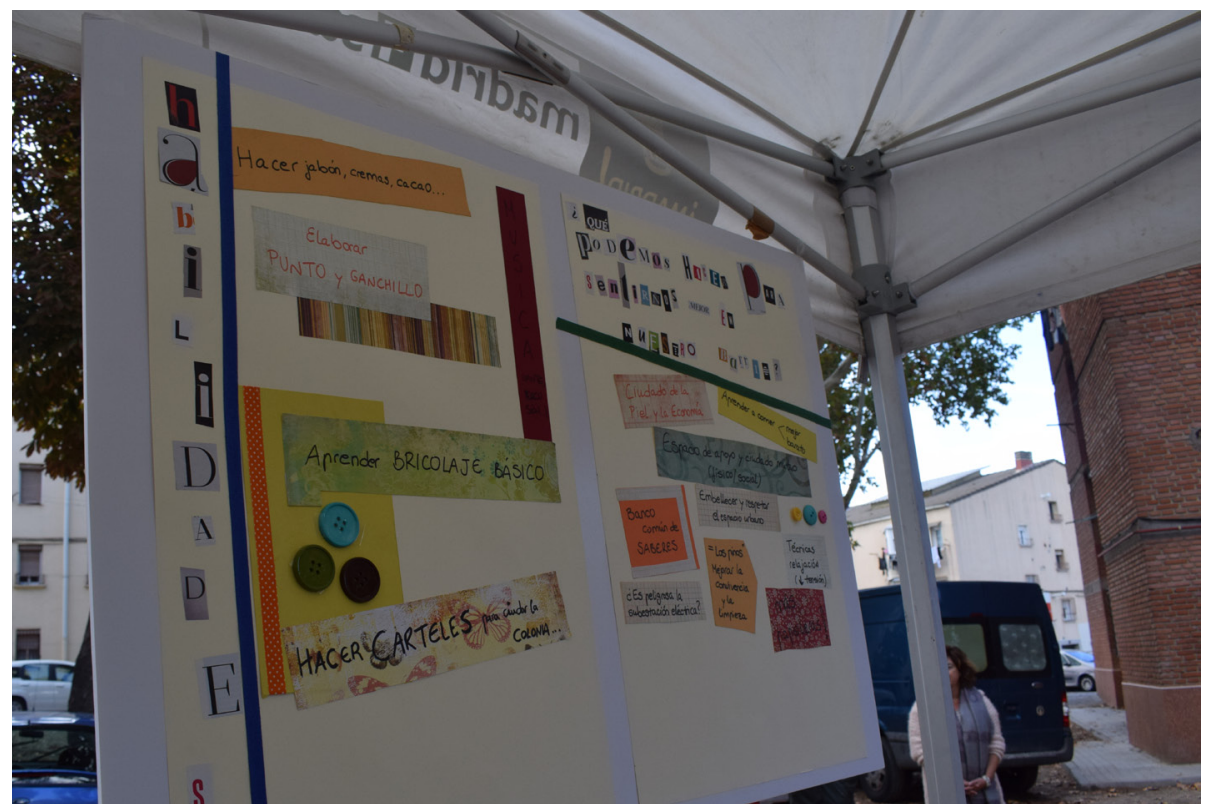

Figura 1. Panel “¿qué podemos hacer en común para sentirnos mejor en nuestro barrio?”.

Por otro lado, la participación ha resultado irregular oscilando entre 30 y 4 personas: la participación en los encuentros y las acciones en el espacio público cuenta con una mayor presencia de personas, sin embargo, en el trabajo de creación colectiva y de organización de las acciones, el número se ha reducido. No obstante, este último aspecto se debe considerar desde el punto de vista cualitativo en términos de la calidad de la participación activa y democrática, ya que, a partir de este momento se consolida un grupo motor que constituye la "locomotora" del proyecto (figura 2) y que irá creciendo en 2018.

Este grupo, no sólo ha llevado a cabo la organización de las acciones y la creación colectiva, sino que también se ha encargado de realizar la evaluación del proceso apuntando varias cuestiones interesantes. Por un lado, manifiestan satisfacción por el trabajo realizado y las acciones desarrolladas, y por otro evidencian las dificultades 
SILES / El arte y la creatividad como nuevas formas de bienestar. Primera fase de ARTYS, La

que, en ocasiones, ha proporcionado el espacio físico de la Colonia Experimental para la instalación artística por la gran cantidad de vehículos aparcados. A su vez, han valorado el trabajo colectivo realizado como un "caos muy productivo y divertido que hay que seguir mejorando dedicando más tiempo al reparto de tareas y a aprender a escuchar", y han evaluado la participación del resto de vecinos y vecinas como "buena, pero mejorable, porque no sé ve lo que hacemos desde fuera".

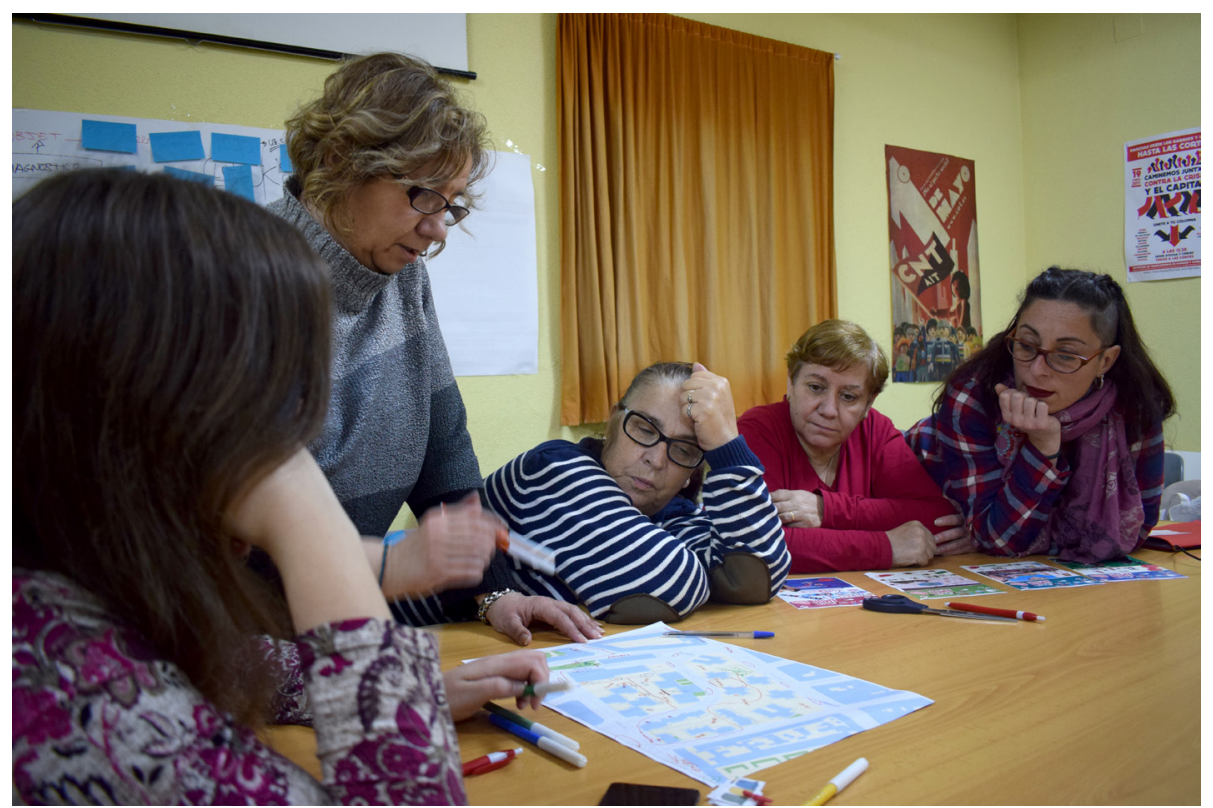

Figura 2. Vecinas del grupo motor de ARTYS. La Experimental.

En relación al arte, se reconoce por parte de las propias participantes, la calidad artística y estética de los carteles con collage (figura 3), mostrando satisfacción por el resultado y queriendo copias para ellas mismas y sus familiares. Igualmente, a través del manifiesto para la acción en el espacio público (figura 4) han expresado comentarios positivos en relación: al carácter colaborativo del proceso de creación observándolo como un "espacio para tejer redes"; al aprendizaje sobre arte, valorando la posibilidad de "acceder al arte desde lo cotidiano"; a la mirada sobre ellas mismas como artistas, soñando con "exponer en el MoMA de Nueva York"; y al reto creativo que supone la creación misma de los carteles con unos lenguajes que eran totalmente ajenos y que se han incorporado en su trabajo habitual dentro del proyecto.

Este documento (figura 4) constituye un producto artístico a la vez que una herramienta creativa de recogida de datos a través de 3 preguntas sencillas en apariencia, pero que han sido claves para recopilar gran cantidad de información relevante: ¿Qué es ARTYS?, ¿cómo nos sentimos?, ¿y el futuro? Se respondieron las preguntas de manera colectiva y ha constituido una primera evaluación del impacto de las prácticas artísticas en la mejora del bienestar de las vecinas y los vecinos participantes 
SILES / El arte y la creatividad como nuevas formas de bienestar. Primera fase de ARTYS, La Experimental, proyecto de Arte y Salud Comunitaria en la Colonia Experimental de Villaverde Alto (Madrid)

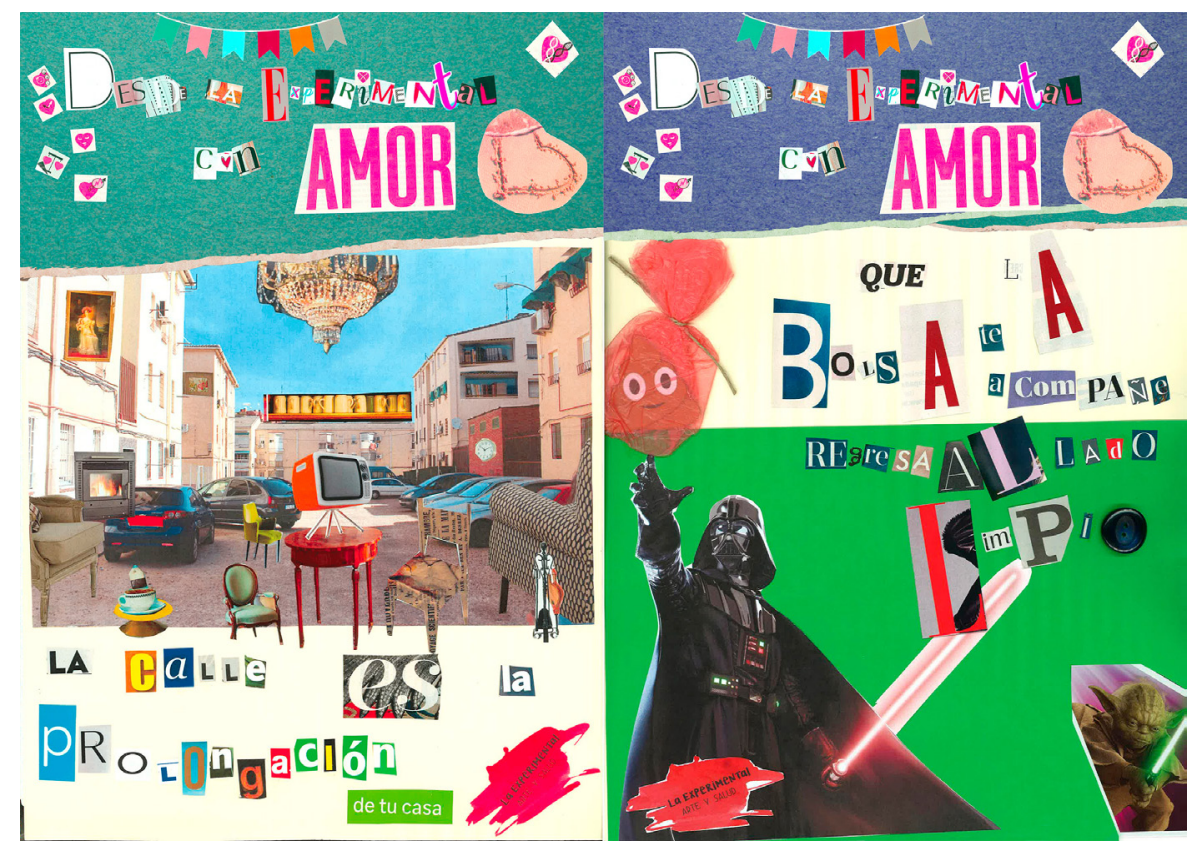

Figura 3. Carteles producidos en el taller "Del tema al lema". ARTYS. La Experimental.

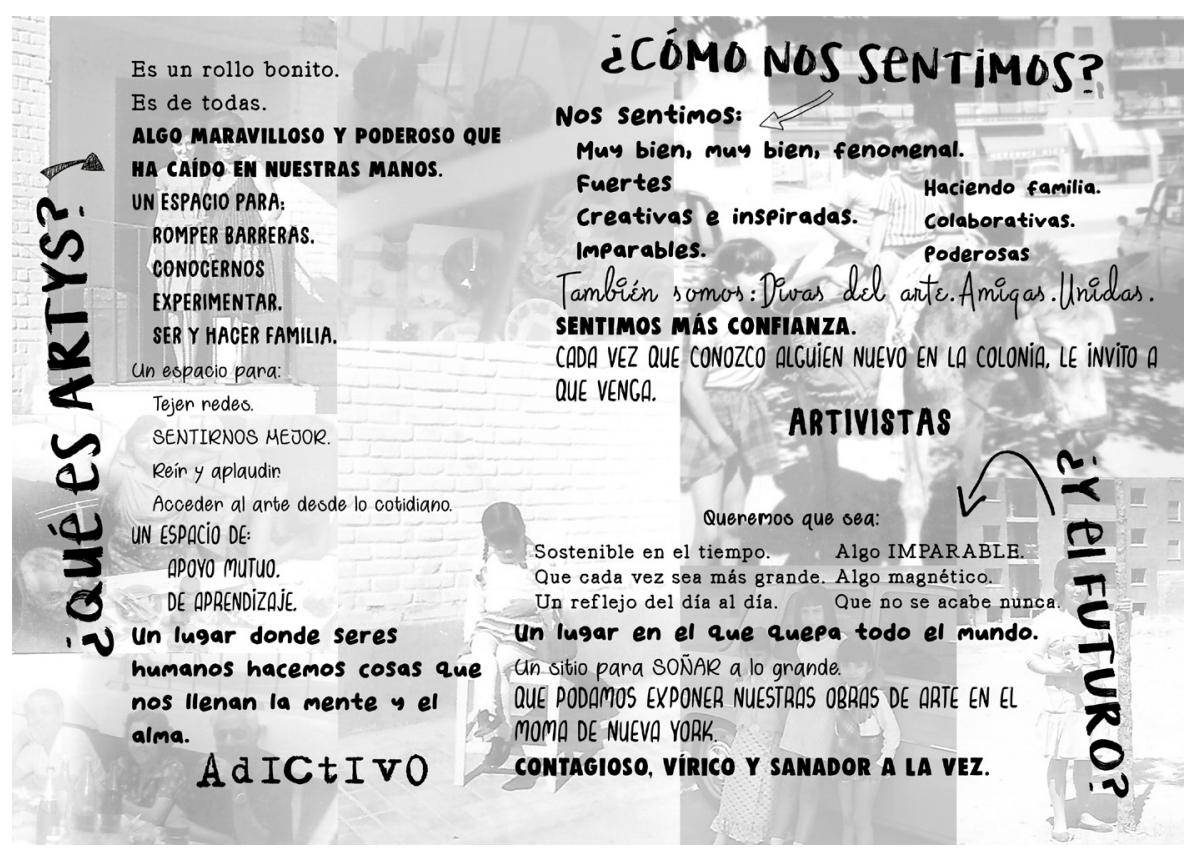

Figura 4. Manifiesto de ARTYS. La Experimental.

Asimismo, desde el punto de vista de la salud comunitaria, en este mismo manifiesto (figura 4) reflejan cómo se sienten participando en este proyecto: "muy bien, fuertes, imparables, poderosas, haciendo familia, amigas, unidas y sienten más confianza". Estas palabras recogen las cuestiones de participación, relaciones comunitarias, creación de vínculos y propias capacidades que son de suma importancia en la promoción de la salud comunitaria de enfoque salutogénico y que se reflejan en los objetivos del proyecto. 
SILES / El arte y la creatividad como nuevas formas de bienestar. Primera fase de ARTYS, La

Por último, cabe mencionar que, durante la acción en el espacio público se ocupó una porción de este, que normalmente es ocupada por vehículos, para instalar un dispositivo artístico que permaneció dos semanas en el mismo lugar, al igual que los carteles. Ante estos hechos, las vecinas han manifestado sentir que han "conquistado a los coches una parcela del espacio para la comunidad" (figura 5).

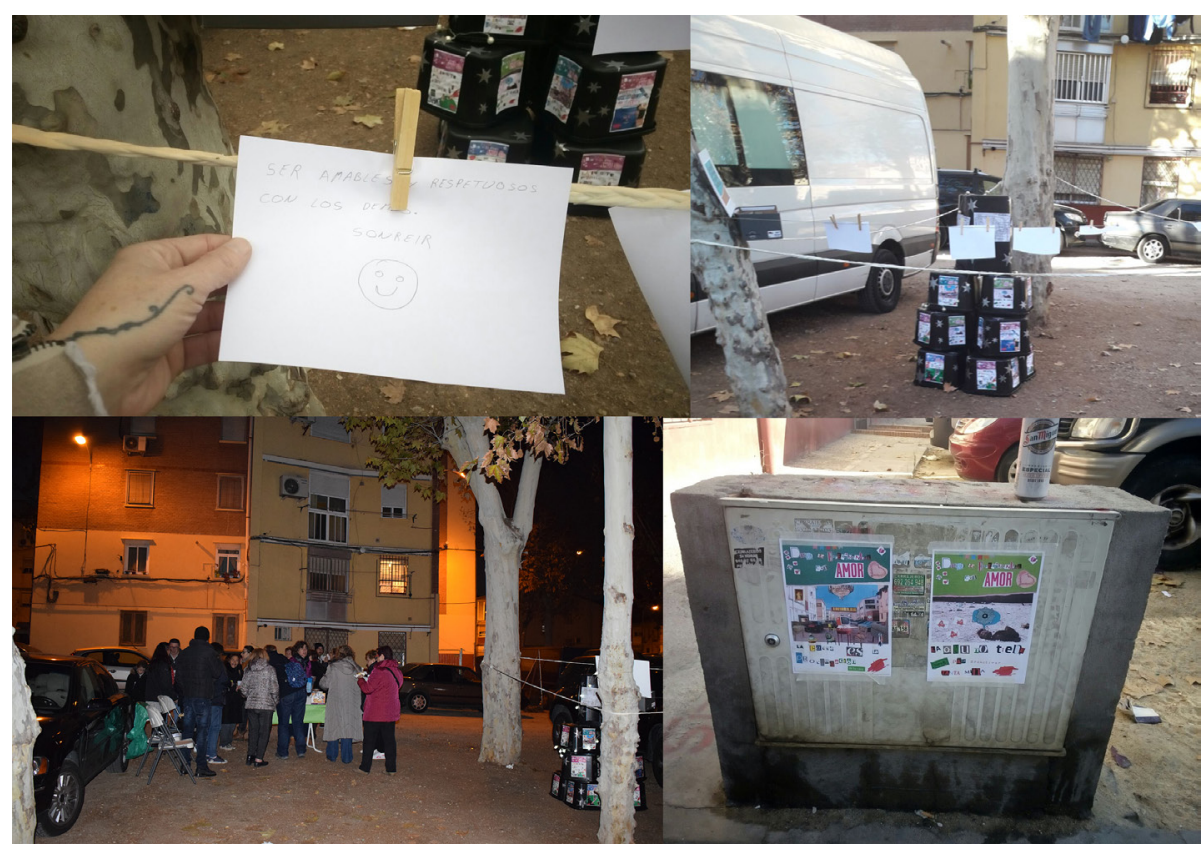

Figura 5. Carteles e instalación en el espacio público del proyecto ARTYS. La Experimental.

\section{Conclusiones}

Al tratarse de un proyecto de intervención comunitaria, no se traduce en un proyecto finalista, es decir que se trata de un proceso indefinido e inespecífico, que parte del principio que cada comunidad es una realidad particular que tiene que realizar su propio itinerario de desarrollo diferente de cualquier otro. Por lo tanto, será el mismo proceso participativo y comunitario el que vaya a determinar este recorrido, sus finalidades y sus prioridades (Marchioni, Morin Ramírez y Álamo Candelaria, 2013), y cuyos resultados y conclusiones irán construyéndose y cambiando a lo largo de un tiempo abierto que se debe ajustarse a las demandas de la propia comunidad.

Ahora bien, partiendo de esta base, en esta primera fase ya se han puesto sobre la mesa algunas cuestiones enunciadas en los objetivos del proyecto. Así, por ejemplo, las palabras recogidas en el manifiesto (figura 4) en relación a la promoción de la salud comunitaria hacen referencia a sentimientos de bienestar y a cuestiones que aluden al empoderamiento y la autoestima; de manera semejante se mencionan las relaciones personales, comunitarias y los vínculos, así como las cuestiones relacionadas con el arte y al proceso creativo. 
SILES / El arte y la creatividad como nuevas formas de bienestar. Primera fase de ARTYS, La Experimental, proyecto de Arte y Salud Comunitaria en la Colonia Experimental de Villaverde Alto (Madrid)

Del mismo modo, cabe destacar que, cuando se enuncia un futuro "en el que quepa el mundo" (figura 4), se está haciendo referencia a la dificultad para encontrar un espacio público no invadido por coches para colocar los dispositivos artísticos. Este conflicto, que ya surge en la sesión 5, únicamente pudo solventarse con la división del grupo. De tal modo que, mientras una parte del grupo pegaba los carteles, la otra "hacía guardia" a la espera de poder "conquistar" un aparcamiento para la instalación en el mismo momento en el que un hueco quedara libre. De esta manera, aparece por primera vez la imagen de su espacio interbloque como un "parking público" de la que manifiestan, verbalmente, no haber sido conscientes previamente. Es posible que, a partir de estas acciones artísticas, se estén produciendo cambios en las perspectivas de los vecinos y las vecinas en relación a sus necesidades, su entorno y a las propias capacidades para llevar a cabo una "metamorfosis" social que satisfaga dichas necesidades desde la colectividad.

En relación a esta colectividad, tras la participación activa en lo que se puede considerar el primer "microproyecto" de ARTYS La Experimental se forma un grupo motor de personas que pondrán en marcha otras acciones colectivas. Se trata de una "comunidad creativa" que trabaja junta en un proceso artístico común formada por 11 mujeres: 7 vecinas que viven en la Colonia Experimental y alrededores, 2 profesionales socio-sanitarias del CMSc, una profesional del Servicio de Convivencia Intercultural en Barrios y la artista-becaria de Arte y Salud. Esta "comunidad creativa" pondrá en marcha un Banco Común de Conocimiento como práctica artística durante el año 2018 con el objetivo de traducir los posibles aprendizajes que surjan de este nuevo "microproyecto" en obras de arte colectivas en el espacio público.

En definitiva, se podría decir que las prácticas artísticas colaborativas, los métodos del arte basado en la comunidad y del arte activista están permitiendo aprender nuevas formas de ver, sentir, elegir y vivir, a través de un proceso democrático y compartido. En él, se tienen en cuenta las opiniones de las personas que participan, sus decisiones, sus lenguajes y sus múltiples formas de expresión creativa para formar una "comunidad-autora" de las manifestaciones plásticas, visuales, simbólicas y performáticas resultado de este tipo de proyectos. Sin embargo, para que todo esto ocurra se considera fundamental que los procesos se planteen a largo plazo. Este planteamiento permitiría adquirir los aprendizajes necesarios que permitan superar las dificultades propias del trabajo colectivo a través del arte. Así, por ejemplo, se estima importante contar con tiempo para superar la falta de confianza que, en ocasiones, se presenta ante la novedad de los lenguajes artísticos, las barreras comunicativas y en el acceso compartido de la información, la tendencia a la monopolización del discurso por parte de algún miembro del grupo, la puesta en práctica de la escucha activa y la renegociación del proyecto a lo largo de todo el desarrollo del trabajo. 
SILES / El arte y la creatividad como nuevas formas de bienestar. Primera fase de ARTYS, La Experimental, proyecto de Arte y Salud Comunitaria en la Colonia Experimental de Villaverde Alto

\section{Referencias}

Blanco, F., El Banquete, DosJotas. (2016). All together now? Jornadas sobre prácticas artísticas colaborativas. Seminario de debate en Medialb Prado. Madrid, España: Recuperado de http://www.hablarenarte.com/catalogos/doc_glosario_ imposible/contexto.pdf

Cofiño, R., Aviñó, D., Benedé C. B., Botello, B., Cubillo, J., Morgang M., Hernán, M. (2016). Promoción de la salud basada en activos: ¿cómo trabajar con esta perspectiva en intervenciones locales? Gaceta sanitaria. Sociedad Española de Salud Pública y Administración Sanitaria. Informe SESPAS, 30(S1), 93-98.

Delgado, M. (2013). Artivismo y pospolítica. Sobre la estetización de las luchas sociales en contextos urbanos. Quaderns-e. Institut Catalá d'Antropologia, 2(18), 68-80.

Fernández Quesada, B. (2004). Nuevos lugares de intención. Intervenciones artísticas en el espacio urbano como una de las salidas a los circuitos convencionales. Estados Unidos 1965-1995 (tesis doctoral). Universitat de Barcelona, España.

Giménez Romero, C. (2013). Promoviendo la convivencia ciudadana intercultural en barrios de alta diversidad. Ideas y experiencias para una práxis comunitaria. En Bauades, J., y Giménez Romero, C. (Coords.). (2013). Hagamos de nuestro barrio un lugar habitable. Manual de intervención comunitaria en barrios (pp. 36-58). Valencia, España: Tirant Humanidades.

Hernán, M., Morgan A., y Mena A. L. (2010). Formación en salutogénesis y activos en salud. Serie Monografías EASP $\mathrm{n}^{\circ}$ 15. CIUDAD Y PAIS Escuela Andaluza de Salud Pública. Consejería de Salud y Bienestar Social. Recuperado de https://www. easp.es/project/formacion-en-salutogenesis-y-activos-para-la-salud/

Lewin, K. (2006). La Investigación Acción y los Problemas de las minorías. En Salazar, M. C., (coord..), (2006), La Investigación-Acción Participativa. Inicios y Desarrollos (pp. 15-27). Caracas, Venezuela: Editorial Laboratorio Educativo.

López Ruíz, V., y Padilla, J., (coords.). (2017). Salubrismo o barbarie. Córdoba, España: Atrapasueños editorial. Madrid Salud. Centros Municipales de Salud Comunitaria.

Marchioni, M., Morin, L. M., y Álamo, J. (2013). Metodología de la intervención comunitaria. Los procesos comunitarios. En Bauades, J., y Giménez Romero, C. (Coords.). (2013). Hagamos de nuestro barrio un lugar habitable. Manual de intervención comunitaria en barrios (pp. 58-74). Valencia, España: Tirant Humanidades. 
SILES / El arte y la creatividad como nuevas formas de bienestar. Primera fase de ARTYS, La Experimental, proyecto de Arte y Salud Comunitaria en la Colonia Experimental de Villaverde Alto (Madrid)

Miyagui, J. (2014). Museo Itinierante "Arte por la Memoria. Parte 3: Experiencias de Activismo Cultural: aprendizajes. En Salazar, X., y Olivos, F. (eds.), Artivismo. Cambio social y activismo cultural. Seminario de debate llevado a cabo en el Instituto de Estudios en Salud, Sexualidad y Desarrollo Humano de la Universidad Peruana Cayetano Heredia, Lima, Perú.

Morgan, A., Davis, M., y Ziglio, E. (2010). Health assets in a global context: theory, methods, action. Nueva York, Estados Unidos: Springer.

Observatorio del tercer Sector Bizkaia. (2016). La Metodología Oasis: una propuesta viva de construcción. Oasis Bizkaia.

Olivos, F. (2014). Proyecto Ciudadaníasx: activismo cultural y derechos humanos. En: Salazar, X. y Olivos, F. (eds.), (2014), Artivismo. Cambio social y activismo cultural. Seminario de debate llevado a cabo en el Instituto de Estudios en Salud, Sexualidad y Desarrollo Humano de la Universidad Peruana Cayetano Heredia, Lima, Perú.

Palacios, A. (2009). El arte comunitario: origen y evolución de las prácticas artísticas colaborativas. Arteterapia: Papeles de arteterapia y educación artística para la inclusión social, 4, 197-211.

Pasarín M. I. y Díaz E. (2013). Salud Comunitaria: una actuación necesaria. Gaceta Sanitaria, 27(6), 477-478.

Rivera de los Santos, F., Ramos, P., Moreno, C., y Hernán, M. (2011). Análisis del modelo salutogénico en España: aplicación en salud pública e implicaciones para el modelo de activos en salud. Revista Española de Salud Pública, 85 (2). 129-139.

Ruiz Peralta, E. (2017). Movimientos sociales y Salud. En López Ruíz, V., y Padilla, J., (coords.), (2017). Salubrismo o barbarie. Córdoba: Atrapasueños editorial.

Radulescu, M. (2014). Movilización y cambio social. Experiencias y lecciones de artivismo cultural: Un enfoque semiótico del arte en el activismo cultural. En: Salazar, X. y Olivos, F. (eds.). (2014). Artivismo. Cambio social y activismo cultural. Seminario de debate llevado a cabo en el Instituto de Estudios en Salud, Sexualidad y Desarrollo Humano de la Universidad Peruana Cayetano Heredia, Lima, Perú. Recuperado de https://issuu.com/iessdeh/docs/artivismo

Salazar, X. y Olivos, F. (eds.). (2014). Artivismo. Cambio social y activismo cultural. Seminario de debate llevado a cabo en el Instituto de Estudios en Salud, Sexualidad y Desarrollo Humano de la Universidad Peruana Cayetano Heredia, Lima, Perú.

Sánchez de Serdio, A. (2015) Prácticas artísticas colaborativas: comprender, negociar, reconocer, retornar. En Collados, A, y Rodrigo J., (eds.) (2015) Transductores 3. Prácticas artísticas en contexto. Granada, España: Centro José Guerrero. Diputación de Granada. (39-44). 
SILES / El arte y la creatividad como nuevas formas de bienestar. Primera fase de ARTYS, La Experimental, proyecto de Arte y Salud Comunitaria en la Colonia Experimental de Villaverde Alto

Silva, A. (2014). Movilización y Cambio social: Experiencias y lecciones de activismo cultural. En Salazar, X. y Olivos, F. (eds.). (2014), Artivismo. Cambio social y activismo cultural. Seminario de debate llevado a cabo en el Instituto de Estudios en Salud, Sexualidad y Desarrollo Humano de la Universidad Peruana Cayetano Heredia, Lima, Perú.

Un mundo en común. Glosario. México. Recuperado de http://unmundoencomun. com/glosario.html 